\title{
Labyrinthe
}

$5 \mid 2000$

Numéro 5

\section{Norbert Elias ou la sociologie des continuités}

\section{(2) OpenEdition}

1 Journals

Édition électronique

URL : http://journals.openedition.org/labyrinthe/273

DOI : 10.4000/labyrinthe. 273

ISSN : 1950-6031

Éditeur

Hermann

Édition imprimée

Date de publication : 15 janvier 2000

Pagination : 89-95

Référence électronique

"Norbert Elias ou la sociologie des continuités », Labyrinthe [En ligne], 5 | 2000, mis en ligne le 17

février 2005, consulté le 20 avril 2019. URL : http://journals.openedition.org/labyrinthe/273; DOI

$10.4000 /$ labyrinthe. 273

Ce document a été généré automatiquement le 20 avril 2019.

Propriété intellectuelle 


\section{Norbert Elias ou la sociologie des continuités}

\section{NOTE DE L'ÉDITEUR}

Roger Chartier nous a révélé l'existence d'un texte introuvable depuis quinze ans ; Kristina Schulz, chercheur à l'université de Bielefeld, en assure le commentaire... Cette introduction à plusieurs voix, inédite et originale, à la grande œuvre d'Elias, inaugure la rubrique « Documents » de Labyrinthe.

(M. Rigo)

Entretien paru en premier lieu le 5 décembre 1985: Roger Chartier l'a assuré et traduit pour le compte du quotidien Libération, que Labyrinthe remercie pour son autorisation à la publication. (Sauf indication contraire, les notes qui suivent sont de Kristina Schulz).

1 La rencontre parisienne entre Norbert Elias et Roger Chartier, en cet hiver 1985, n'est pas leur premier contact : la rencontre intellectuelle est antérieure. Les questions posées durant l'entretien témoignent de la familiarité de Roger Chartier avec les concepts et modèles de son " aîné » de 48 ans. C'est en effet à partir du milieu des années quatrevingts qu'il découvre et introduit l'œuvre d'Elias dans l'historiographie française : ce document en témoigne.

2 La réception internationale de l'œuvre de Norbert Elias est en effet tardive : alors que la plupart de ses grandes analyses datent des années trente, sa lecture, en France comme ailleurs, ne commence que trois décennies plus tard. C'est La Société de cour, paru en France en 1974, qui attire en premier lieu l'attention de Roger Chartier. Le livre, écrit entre 1930 et 1933 alors que Norbert Elias est assistant de Karl Mannheim à l'université de Francfort-sur-le-Main, intéresse celui qui est alors le promoteur de l'histoire culturelle de l'Ancien Régime et de la Révolution. Cet objet commun d'analyse n'est pas la seule raison 
de son intérêt, également méthodologique, comme en témoigne l'avant-propos qu'il donne à la réédition de 1985 .

3 Au-delà du concept central de « configuration ", qu'on retrouvera dans l'entretien, Roger Chartier s'arrête sur les notions d'« interdépendance » et d'« équilibre des tensions », qui caractérisent une approche et une conception de l'homme fondée sur l'idée que "ce qu'on a coutume de désigner par deux concepts différents, [l'individu et la société], ne constituent pas, comme l'emploi actuel de ces deux termes nous le fait souvent croire, deux objets d'existence séparée : ce sont des niveaux différents mais inséparables de l'univers humain ${ }^{1}$ ".

4 Fidèle à son amour éliasien, Roger Chartier préface dans les années quatre-vingt-dix les textes d'Elias parus chez Fayard : La Société des individus, Engagement et Distanciation, et Sport et Civilisation. Son grand essai sur Les Origines culturelles de la Révolution française ("L'univers historique », Seuil, 1990) est imprégné de l'analyse du sociologue allemand, dans La Dynamique de l'Occident, d'un processus de très longue durée, du Moyen Âge au XVIII ${ }^{e}$ siècle, de maîtrise de la violence. Roger Chartier s'essaie à déterminer les relations entre ce processus d'apaisement progressif, et l'émergence soudaine de la violence révolutionnaire, posant la question majeure des rapports de la continuité et de la rupture, facteur de polarisation encore très actuel dans la communauté des historiens, pour qui l'opposition des structures et de l'événement semble souvent insurmontable.

5 Norbert Elias - et Roger Chartier dans son sillage - met au centre de son questionnement cette tension de deux modes de temporalité : il est important, dans un moment historiographique comme le nôtre où l'histoire événementielle paraît l'avoir emporté sur l'histoire des structures, de rappeler que ces moyens conceptuels existent, et que toute " constellation " historique, quand même les contemporains la percevraient comme unique, est à étudier comme tributaire d'un développement antérieur. Cette leçon, fût-elle devenue celle d'un historien comme Chartier, a-t-elle aujourd'hui encore reçu tous ses développements?

7 «La visite à Paris de Norbert Elias, c'est d'abord une image. Celle d'un vieux monsieur de quatrevingt-huit ans qui entre à petits pas dans une salle du Collège de France, s'accoude au bureau et qui, pendant une heure, debout, sans une faiblesse, traverse l'histoire du savoir comme il a traversé l'histoire intellectuelle de son siècle ${ }^{2}$.

8 Deux jours plus tard, à l'École des Hautes Études en Sciences Sociales, son séminaire était consacré aux transformations des relations entre les sexes - et cette fois encore le parcours enjambe le temps, de la Grèce archaïque à la Vienne de 1900, de la Rome des empereurs à aujourd'hui, pour repérer, dans une évolution de très longue durée, les figures du changement. Ainsi a pu être prise une nouvelle mesure de l'extraordinaire vitalité de cet homme qui quittal'Allemagne de Hitler dès le printemps de 1933, s'installa en Angleterre deux ans plus tard, fut pofesseur à l'Université de Leicester entre 1954 et sa retraite en 1962, qui a donné et poursuit encore l'une des œuvres majeures de notre époque, enfin lue, comprise et retenue ${ }^{3}$.

9 Nous avons pris appui sur ces deux textes dans lesquels Norbert Elias a fait une place inattendue à l'Antiquité, de Sumer à Rome, et aussi sur la republication en poche de sa Société de cour (Flammarion, Champs) dans une édition qui comprend l'avant-propos très anti-historien écrit en 1969 pour l'édition allemande, pour l'interroger sur ses relations avec Freud, Kant, les Grecs et les historiens. Voici ses réponses. 

d'oppositions statiques. Nous tenons l'innovation comme absolument incompatible avec la continuité alors qu'il est clair, par exemple, que les avancées intellectuelles faites dans la Grèce ancienne n'auraient pu l'être sans le savoir reçu des Babyloniens. Mais nous pensons encore ethnocentriquement, et nous nous identifions avec les Grecs, pas avec les Babyloniens, avec l'Occident, pas avec l'Orient. Nos concepts reflètent cette identification qu'il ne faut pas projeter dans les faits eux-mêmes. Les sciences de la nature ont imposé le modèle des relations de cause à effet, mais nous ne pouvons pas rendre compte du rapport entre les Babyloniens et les Grecs en termes de continuité. Il faut rétablir une correcte compréhension des continuités en masquant les découvertes et les innovations dans l'ensemble des conditions, antérieures et obligées, qui les ont rendues possibles. C'est pourquoi je voudrais substituer la notion de développement du savoir à celle de « révolution scientifique ", pourquoi aussi je me sens tout à fait séparé de théories comme celles de Kuhn et Popper qui brisent la nécessaire continuité entre ce qu'elles distinguent comme « préscientifique » et ce qu'elles tiennent pour scientifique en universalisant notre propre conception de la science.

12 L. - Comment cet accent porté sur les continuités dans la constitution du savoir s'articule-t-il avec un des concepts centraux de votre œuvre, celui de figuration ${ }^{4}-$ que l'on peut rendre en français par formation ou, mieux, configuration - et qui entend marquer les fondamentales différences qui contrastent les formes sociales, les manières de penser, les structures psychiques?

13 N.E. - Ce concept de figuration a souvent été mal compris. Pour moi, il s'agit d'une notion essentiellement dynamique, et point du tout statique, qui permet de comprendre le passage d'une situation à une autre.

14 Laissez-moi prendre deux exemples. Dans mon livre, traduit en français sous le titre La Dynamique de l'Occident, j'ai analysé comment en france aux $\mathrm{XVI}^{\mathrm{e}}$ et $\mathrm{XVII}^{\mathrm{e}}$ siècles la noblesse féodale et guerrière devenait progressivement une aristocratie de cour, domestiquée et pacifique ; il y a bien là un changement, mais un changement opéré à travers un processus de longue durée qui recompose de manière inédite la formation sociale tout entière. Autre exemple : à Rome, entre 400 avant J.-C. et 100 après J.-C., les rapports entre hommes et femmes se trouvent profondément modifiés, substituant la plus large égalité (au moins pour les femmes mariées) à la plus brutale inégalité. Là encore le changement doit être compris, non comme une discontinuité ou une rupture, mais comme un processus dynamique de transformation de la configuration sociale, qui fait passer d'un état à un autre.

15 L. - Vous avez toujours fortement marqué la différence existant entre votre approche sociologique et celle des historiens. Le fait que les historiens ne travaillent plus aujourd'hui sur les mêmes objets que leurs prédécesseurs vous a-t-il conduit à modifier votre appréciation?

16 N.E. - En aucune manière. Je pense que les historiens continuent àtravailler à l'intérieur d'un découpage chronologique rigide qui crée d'arbitraires discontinuités, mais qui est sans doute nécessaire pour un travail de spécialiste. Ma perspective est autre : elle vise à transcender ces frontières canoniques, ces cloisonnements chronologiques, pour 
comprendre dans leur très longue durée les processus qui ont permis les avancées du savoir ou ceux qui ont modifié les attitudes et les comportements des hommes les uns envers les autres. Bien sûr, je ne pourrais pas faire ce que je fais sans les travaux des historiens mais le sociologue, qui n'est pas enfermé dans les périodisations classiques, peut développer une compréhension synthétique d'un plus haut degré. L. - Pourquoi portez-vous maintenant si grand intérêt à l'Antiquité et que signifie pour vous ce retour aux Anciens, fait aussi par Michel Foucault dans ses derniers livres? N.E. - L'Antiquité n'est pas une chose morte appartenant au passé mais elle conditionne nos propres manières de penser. J'ai été très heureux de voir que Foucault, après ses livres trop spéculatifs et abstraits à mon goût, s'était attaché à comprendre le développement de la conscience ou de l'image de soi chez les Grecs - je dirais plutôt, dans mon vocabulaire, les formations de la structure la personnalité. L'important est de reconstituer la démarche de très longue durée qui a conduit à notre actuelle définition de la connaissance et à la structure de la personnalité qui est la nôtre. Les Grecs n'avaient pas vraiment de mots pour désigner la conscience individuelle ou la relation de causalité. Il nous faut donc comprendre ce "non-savoir" pour comprendre comment le nôtre a pu se constituer.

L. - Vous parlez de "structure de la personnalité". Pouvez-vous préciser quelle est votre relation à l'œuvre de Freud et votre appréciation des concepts majeurs de la psychanalyse ?

N.E. - Sans Freud, je n'aurais pas pu écrire ce que j'ai écrit. Sa théorie a été essentielle pour mon travail et tous ses concepts (moi, surmoi, libido, etc.) me sont très familiers. Mais Freud, sa vie durant, a étudié les hommes et les femmes qui vivaient à la fin du XIX ${ }^{\mathrm{e}}$ siècle et au début $\mathrm{du} \mathrm{XX}^{\mathrm{e}}$ siècle et, à la manière des sciences de la nature, il a forgé ses concepts comme si la structure de la personnalité qu'il observait était celle de tous les hommes humains. Dans Totem et Tabou, il perçoit que ce n'est peut-être pas le cas, mais il dut tellement batailler pour faire reconnaître la légitimité de son enseignement qu'il donna en fait valeur universelle à un type donné et daté de structures de la personnalité. Aller au-delà de Freud, scientifiquement parlant, c'est reconnaître les transformations qui affectèrent le développement de la personnalité humaine et penser que dans un monde où le savoir sur le monde naturel n'était pas le nôtre, où les peurs et anxiétés étaient obsédantes, la structure de la personnalité ne peut être celle des hommes du XXe siècle. D'où la nécessité d'employer d'autres termes et d'autres concepts que ceux de Freud pour caractériser les économies psychiques anciennes 5 . Par exemple on ne peut pas parler d'un surmoi de l'homme médiéval. Le problème est de comprendre comment et pourquoi émergea progressivement la structure de la personnalité qui est décrite par Freud.

L. - Que pensez-vous du retour à Kant et de la vogue, en France et ailleurs, du néokantisme?

N.E. - Je pense, à partir de l'examen des faits, et non par simple opinion, qu'il n'existe rien de semblable à ce que Kant a conceptualisé spéculativement avec les catégories d'a priori et de transcendantal - c'est-à-dire postulant l'existence de formes de pensée innées. J'ai indiqué à plusieurs reprises que si Kant avait pu considérer la raison comme donnée à nous par la nature, c'est parce qu'à son époque la biologie n'était pas suffisamment développée pour donner au terme nature un sens purement biologique. Il me parait absurde de penser que des concepts comme "cause", "nature", "lois naturelles", et d'autres, sont donnés de manière innée à des êtres humains. Ce n'est donc pas moi mais 
les faits qui montrent qu'a priori les limites transcendantales de la connaissance n'existent pas.

L. - Quelles sont les raisons qui vous ont conduit à étudier les relations changeantes et les équilibres instables entre les sexes?

N.E. - Si je suis intéressé par le passé, je suis aussi profondémentconcerné par les questions de mon époque. Et je suis sûr que les changements des relations entre les sexes sont l'un des principaux bouleversements de notre temps. J'ai eu l'expérience, au cours de ma longue vie, de cette transformation entre les manières dont les hommes et les femmes vivent ensemble. Celle d'aujourd'hui aurait été impensable au début de ce siècle. Pour comprendre cette évolution, je me suis attaché à d'autres périodes qui, elles aussi, ont connu de semblables changements - par exemple à Rome entre le III siècle avant J.-C. et le $\mathrm{I}^{\mathrm{er}}$ siècle après $\mathrm{J}$.-C. L'époque même dans laquelle je vis m'impose certaines questions qu'il faut situer dans le long terme de "figurational changes", c'est-à-dire le long terme des formes sociales et des dispositifs psychologiques. »

\section{NOTES}

1. N. Elias, Qu'est-ce que la sociologie ?, Paris, Agora, 1991, p. 156.

2. Norbert Elias est né en 1897 à Breslau et mort en 1990 à Amsterdam.

3. L'accès tardif à l'œuvre de Norbert Elias se comprend si l'on étudie de près la chronologie de la publication, révélant un grand écart entre la rédaction des textes et leur parution sous forme de livres : pratique éditoriale due à des circonstances tant historiques que biographiques. La Société des individus, paru en allemand en 1987, comporte par exemple trois textes de 1939 («La Société des individus »), des années 40-50 ( Conscience de soi et image de l'homme »), et de 1986-1987 (« Les transformations de l'équilibre "nous-je” »). À cela s'ajoute le délai de traduction : pour Sport et Civilisation, paru en allemand en 1986, la parution française est de 1994.

4. « Une figuration est une formation sociale dont la taille peut être fort variable [...] où les individus sont liés les uns aux autres par un mode spécifique de dépendances réciproques et dont la reproduction suppose un équilibre mobile de tensions. » (Roger Chartier, Introduction à La Société de cour, p. X). Elias illustre cette notion de figuration (" configuration » ou «formation ») par l'image du jeu : « Quatre hommes assis autour d'une table pour jouer aux cartes forment une configuration. Leurs actes sont interdépendants [...] Ce qu'il faut comprendre par configuration, c'est la figure globale toujours changeante que forment les joueurs ; elle inclut non seulement leur intellect mais toute leur personne, les actions et les relations réciproques [...]. L'interdépendance des joueurs, condition nécessaire à l'existence d'une configuration spécifique, est une interdépendance d'alliés mais aussi d'adversaires. » (Norbert Elias, Qu'est-ce que la sociologie ?, p. 157). À l'intérieur d'une formation, le but est dans cette perspective de « saisir les particularités de ces configurations complexes par l'analyse des chaînes d'interdépendance. » (ibid., p. 159). 
5. Norbert Elias conçoit l'individu comme existence interdépendante, qui ne trouve son sens que dans une formation concrète constituée de l'ensemble des individus. C'est cet ensemble qui détermine les contraintes sociales (ou mieux peut-être « formationnelles ») auxquelles les individus sont soumis et qu'ils intériorisent. Il s'agit non seulement des contraintes économiques ou sociales au sens courant, restreint, mais aussi de celles qui concernent la vie des pulsions et des émotions : «le processus de civilisation » se caractérise justement par l'acquisition progressive du contrôle de cette vie émotionnelle. L'économie psychique, dans cette perspective, n'est pas un état fixe caractérisé selon les topiques freudiennes, mais une étape, limitée, d'un processus dont il s'agit de déterminer les caractéristiques spécifiques. 